\title{
EFFECTS OF TEMPERATURE DEPENDENT THERMAL CONDUCTIVITY AND VISCOUS DISSIPATION ON FREE CONVECTIVE FLOW ALONG A VERTICAL FLAT PLATE WITH ASYMPTOTIC SOLUTION
}

\author{
A. K. M. Safiqul Islam ${ }^{1, *}$, M. A. Alim ${ }^{2}$, Md. Rezaul Karim ${ }^{3}$ and ATM. M. Rahman ${ }^{4}$ \\ ${ }^{1}$ Department of Mathematics, Gowripur Govt. College, Mymensingh, Bangladesh. \\ ${ }^{2}$ Department of Mathematics, Bangladesh University of Engineering and Technology, \\ Dhaka-1000, Bangladesh. \\ ${ }^{3}$ Department of Mathematics, Jagannath University, Dhaka-1100, Bangladesh \\ ${ }^{4}$ Department of Computer Science and Engineering, Dhaka International University, \\ Dhaka-1213, Bangladesh. \\ "Corresponding author: asafiqis@gmail.com
}

Received 06.08.2015 Accepted 04.08.2016

\begin{abstract}
This paper reports us the surface temperature distribution effects of free convective flow along a vertical flat plate with temperature dependent thermal conductivity and viscous dissipation with asymptotic solution. The governing equations with associated boundary conditions reduce to local non-similarity boundary layer equations for this phenomenon are converted to dimensionless forms using a suitable transformation. The transformed non-linear equations are then solved using the implicit finite difference method together with Keller-box technique. Numerical results of the velocity and temperature profiles, skin friction and surface temperature profiles for different values of the thermal conductivity variation parameter, the Prandtl number and the viscous dissipation parameters are presented graphically. Also we considered the asymptotic solution for the effect of the thermal conductivity variation parameter, the Prandtl number and the viscous dissipation parameters in skin friction and surface temperature profiles. Detailed discussion is given for the aforementioned parameters. A good similarity is found in small and large value solution with all value solution for the thermal conductivity variation parameter, the Prandtl number and the viscous dissipation parameter for skin friction and surface temperature. Divergence is found near one (1).
\end{abstract}

Keywords: Surface temperature, Small and large values x, Flat plate, Velocity, Prandtl number

\section{Introduction}

Electrically conducting fluid flow in presence of the effect of temperature dependent thermal conductivity flow and heat conduction problems are important from the technical point of view and such types of problems have received much attention by many researchers. In electronics in particular and in physics broadly used both the terms. 
Miyamoto et al. (1980) studied the effect of axial heat conduction in a vertical flat plate on free convection heat transfer. Pozzi and Lupo (1988) investigated the coupling of conduction with laminar convection along a flat plate. Pop et al. (1995) investigated the conjugate mixed convection on a vertical surface in porous medium. Gebhart (1962) investigated the effect of dissipation on natural convection. Takhar and Soundalgekar (1980) studied the dissipation effects on MHD free convection flow past a semi-infinite vertical plate. Khan (2002) investigated the conjugate effect of conduction and convection with natural convection flow from a vertical flat plate and in an inclined square cavity. Mamun (2005) studied the effects of conduction and convection on magnetohydrodynamic flow with and without viscous dissipation from a vertical flat plate. Hossain (1992) analyzed the viscous and Joule heating effects on MHD free convection flow with variable plate temperature. Rahman et al. (2008) investigated the effects of temperature dependent thermal conductivity on MHD free convection flow along a vertical flat plate with heat conduction. Rahman and Alim (2009) analyzed numerical study of MHD free convective heat transfer flow along a vertical flat plate with temperature dependent thermal conductivity. Nasrin and Alim (2009) studied the combined effects of viscous dissipation and temperature dependent thermal conductivity on MHD free convection flow with conduction and joule heating along a vertical flat plate. Alim et al. (2008) analyzed the combined effect of viscous dissipation \& joule heating on the coupling of conduction \& free convection along a vertical flat plate. Alim et al. (2007) investigated Joule heating effect on the coupling of conduction with MHD free convection flow from a vertical flat plate. Chowdhury and Islam (2000) analyzed MHD Free Convection Flow of Visco-elastic Fluid past an Infinite Porous Plate. Alam et al. (2007) studied viscous dissipation effects on MHD natural convection flow over a sphere in the presence of heat generation. Saha et al. (2004) analyzed Natural Convection from a Plane Vertical Porous Surface in Non-Isothermal Surroundings. Islam et al. (2014) investigated Effects of Conduction Variation on MHD Natural Convection Flow Along a Vertical Flat Plate.

The present study is to incorporate the idea of the effects of free convective flow along a vertical flat plate with temperature dependent thermal conductivity and viscous dissipation with asymptotic solution.

\section{Mathematical Formulation of the Problem}

We consider a steady two-dimensional laminar natural convection flow of an electrically conducting, viscous and incompressible fluid along a vertical flat plate of length $l$ and thickness $b$ (Figure-1). It is assumed that the temperature at the outer surface of the plate is maintained at a constant temperature $T_{b}$, where $T_{b}>T_{\infty}$, the ambient temperature of the fluid. In this work $\bar{y}$-axis i.e. normal direction to the surface and $\bar{x}$-axis is taken along the flat plate. The coordinate system and the configuration are shown in Figure-1. 


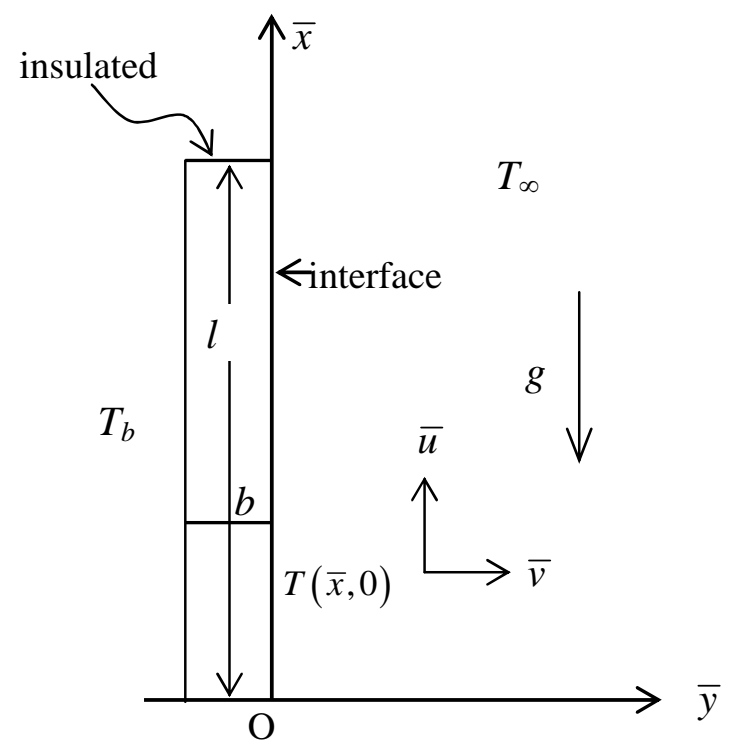

Fig. 1. Physical model and coordinate system

The governing equations of such laminar flow with viscous dissipation and also thermal conductivity variation along a vertical flat plate under the Boussinesq approximations $\rho=\rho_{\infty}\left[1-\beta\left(T_{b}-T_{\infty}\right)\right]$, where $\rho_{\infty}$ and $T_{\infty}$ are the density and temperature respectively outside the boundary layer. For the present problem the continuity, momentum and energy equations take the following forms

$$
\begin{aligned}
& \frac{\partial u}{\partial \bar{x}}+\frac{\partial v}{\partial \bar{y}}=0 \\
& \bar{u} \frac{\partial \bar{u}}{\partial \bar{x}}+\bar{v} \frac{\partial \bar{u}}{\partial \bar{y}}=v \frac{\partial^{2} \bar{u}}{\partial \bar{y}^{2}}+g \beta\left(T_{f}-T_{\infty}\right) \\
& \bar{u} \frac{\partial T_{f}}{\partial \bar{x}}+\bar{v} \frac{\partial T_{f}}{\partial \bar{y}}=\frac{1}{\rho C_{p}} \frac{\partial}{\partial \bar{y}}\left(\kappa_{f} \frac{\partial T_{f}}{\partial \bar{y}}\right)+\frac{v}{C_{p}}\left(\frac{\partial \bar{u}}{\partial \bar{y}}\right)^{2}
\end{aligned}
$$

Here $\beta$ is coefficient of volume expansion. The temperature dependent thermal conductivity, which is used by Rahman (2008) as follows

$$
\kappa_{f}=\kappa_{\infty}\left[1+\delta\left(T_{f}-T_{\infty}\right)\right]
$$

Where $\kappa_{\infty}$ is the thermal conductivity of the ambient fluid and $\delta$ is a constant, defined as $\partial=\frac{1}{\kappa_{f}}\left(\frac{\partial \kappa}{\partial T}\right)_{f}$. The appropriate boundary condition to be satisfied by the above equations are

$$
\begin{aligned}
& \left.\begin{array}{l}
\bar{u}=0, \bar{v}=0 \\
T_{f}=T(\bar{x}, 0), \frac{\partial T_{f}}{\partial \bar{y}}=\frac{\kappa_{s}}{b \kappa_{f}}\left(T_{f}-T_{b}\right)
\end{array}\right\} \text { on } \bar{y}=0, \bar{x}>0 \\
& \bar{u} \rightarrow 0, T_{f} \rightarrow T_{\infty} \text { as } \bar{y} \rightarrow \infty, \bar{x}>0
\end{aligned}
$$


The non-dimensional governing equations and boundary conditions can be obtained from equations (1) - (3) using the following dimensionless quantities

$$
\begin{aligned}
& x=\frac{\bar{x}}{l}, \quad y=\frac{\bar{y}}{l} G r^{\frac{1}{4}}, u=\frac{\bar{u} l}{v} G r^{-\frac{1}{2}}, v=\frac{\bar{v} l}{v} G r^{-\frac{1}{4}}, \\
& \theta=\frac{T_{f}-T_{\infty}}{T_{b}-T_{\infty}}, G r=\frac{g \beta l^{3}\left(T_{b}-T_{\infty}\right)}{v^{2}}
\end{aligned}
$$

Where $l$ is the length of the plate, $G r$ is the Grashof number, $\theta$ is the dimensionless temperature.

Now from equations (1)-(3), we get using the following dimensionless equations

$$
\begin{aligned}
& \frac{\partial u}{\partial x}+\frac{\partial v}{\partial y}=0 \\
& u \frac{\partial u}{\partial x}+v \frac{\partial u}{\partial y}=\frac{\partial^{2} u}{\partial y^{2}}+\theta \\
& u \frac{\partial \theta}{\partial x}+v \frac{\partial \theta}{\partial y}=\frac{1}{\operatorname{Pr}}(1+\gamma \theta) \frac{\partial^{2} \theta}{\partial y^{2}}+\frac{\gamma}{\operatorname{Pr}}\left(\frac{\partial \theta}{\partial y}\right)^{2}+N\left(\frac{\partial u}{\partial y}\right)^{2}
\end{aligned}
$$

Where $\operatorname{Pr}=\frac{\mu C_{p}}{\kappa_{\infty}}$ is the Prandtl number, $\gamma=\delta\left(T_{b}-T_{\infty}\right)$ is the non-dimensional thermal conductivity variation parameter and $N=\frac{v^{2} G r}{l^{2} C_{p}\left(T_{b}-T_{\infty}\right)}$ is the dimensionless viscous dissipation parameter. The corresponding boundary conditions (5) then take the following form

$$
\begin{aligned}
& u=0, v=0, \theta-1=(1+\gamma \theta) P \frac{\partial \theta}{\partial y} \text { on } y=0, x>0 \\
& u \rightarrow 0, \theta \rightarrow 0 \text { as } y \rightarrow \infty, x>0
\end{aligned}
$$

Here $P=\left(\frac{\kappa_{\infty} b}{\kappa_{s} l}\right) G r^{\frac{1}{4}}$ is the conduction parameter. In the present investigation we have considered $P=1$.

To solve the equations (8) and (9) subject to the boundary conditions (10) the following transformations are proposed by Merkin \& Pop (1996)

$$
\begin{aligned}
& \psi=x^{\frac{4}{5}}(1+x)^{-\frac{1}{20}} f(x, \eta) \\
& \eta=y x^{-\frac{1}{5}}(1+x)^{-\frac{1}{20}} \\
& \theta=x^{\frac{1}{5}}(1+x)^{-\frac{1}{5}} h(x, \eta)
\end{aligned}
$$

Here $\eta$ is the similarity variable and $\psi$ is the non-dimensional stream function which satisfies the continuity equation and is related to the velocity components in the usual way as 


$$
u=\frac{\partial \psi}{\partial y} \text { and } v=-\frac{\partial \psi}{\partial x}
$$

Moreover, $h(x, \eta)$ represents the non-dimensional temperature. The momentum and energy equations are transformed for the new co-ordinate system. At first, the velocity and temperature components are expressed in terms of the new variables for this transformation. Thus the following equations

$$
\begin{aligned}
& f^{\prime \prime \prime}+\frac{16+15 x}{20(1+x)} f f^{\prime \prime}-\frac{6+5 x}{10(1+x)} f^{\prime 2}+h=x\left(f^{\prime} \frac{\partial f^{\prime}}{\partial x}-f^{\prime \prime} \frac{\partial f}{\partial x}\right) \\
& \frac{1}{\operatorname{Pr}} h^{\prime \prime}+\frac{\gamma}{\operatorname{Pr}}\left(\frac{x}{1+x}\right)^{\frac{1}{5}} h h^{\prime \prime}+\frac{\gamma}{\operatorname{Pr}}\left(\frac{x}{1+x}\right)^{\frac{1}{5}} h^{\prime 2}+\frac{16+15 x}{20(1+x)} f h^{\prime} \\
& -\frac{1}{5(1+x)} f^{\prime} h+N x f^{\prime \prime 2}=x\left(f^{\prime} \frac{\partial h}{\partial x}-h^{\prime} \frac{\partial f}{\partial x}\right)
\end{aligned}
$$

where prime denotes partial differentiation with respect to $\eta$. The boundary conditions as mentioned in equation (10) then take the following form

$$
\begin{aligned}
& f(x, 0)=f^{\prime}(x, 0)=0 \\
& h^{\prime}(x, 0)=\frac{x^{\frac{1}{5}}(1+x)^{-\frac{1}{5}} h(x, 0)-1}{(1+x)^{-\frac{1}{4}}+\gamma x^{\frac{1}{5}}(1+x)^{-\frac{9}{20}} h(x, 0)} \\
& f^{\prime}(x, \infty) \rightarrow 0, h(x, \infty) \rightarrow 0
\end{aligned}
$$

Secondly, from the process of numerical computation, in practical point of view, it is important to calculate the values of the surface shear stress in terms of the skin friction coefficient. This can be written in the non-dimensional form as Molla et al. (2005)

$$
C_{f}=\frac{G r^{-\frac{3}{4}} l^{2}}{\mu v} \tau_{w}
$$

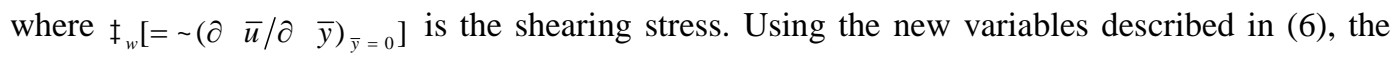
local skin friction coefficient can be written as

$$
C_{f x}=x^{\frac{2}{5}}(1+x)^{-\frac{3}{20}} f^{\prime \prime}(x, 0)
$$

In practical point of view, it is important to calculate the values of the surface temperature. The numerical values of the surface temperature are obtained from the relation. This can written in the non-dimensional form as

$$
\theta(x, 0)=x^{\frac{1}{5}}(1+x)^{-\frac{1}{5}} h(x, 0)
$$

We have also discussed the velocity and the temperature profiles for different values of the thermal conductivity variation parameter, Prandtl number and viscous dissipation parameter. 


\section{Numerical Method of Solution}

In this paper investigates the effect of surface temperature distribution for the temperature dependent thermal conductivity and viscous dissipation on electrically conducting fluid in natural convection flow along a vertical flat plate with asymptotic solution. Along with the boundary conditions (14), the solution of the parabolic non-linear ordinary differential equations (12) and (13) will be found by using the implicit finite difference method together with Keller-box elimination technique or scheme (1978) which is well documented by Cebeci and Bradshaw (1984) and widely used by Keller and Cebeci and Hossain (1992).

\section{Asymptotic Solution}

Now, we have given attention to the behavior of the solution of the equations (12) and (13) when $x$ is positively small (for $x<1)$ and large $\left(\frac{1}{x}\right.$ as $\left.x \rightarrow 0\right)$ for $\gamma, \operatorname{Pr}$ and $N$. Also we need the solution of the equations (16) and (17) when $x$ is small and large for $\gamma, \operatorname{Pr}$ and $N$.

As we see, for small $x$ equations (12) and (13) become:

$$
\begin{aligned}
& f^{\prime \prime \prime}+\frac{4}{5} f f^{\prime \prime}-\frac{3}{5} f^{\prime 2}+h=x\left(f^{\prime} \frac{\partial f^{\prime}}{\partial x}-f^{\prime \prime} \frac{\partial f}{\partial x}\right) \\
& \frac{1}{\operatorname{Pr}} h^{\prime \prime}+\frac{4}{5} f h^{\prime}-\frac{1}{5} f^{\prime} h+N x f^{\prime \prime 2}=x\left(f^{\prime} \frac{\partial h}{\partial x}-h^{\prime} \frac{\partial f}{\partial x}\right)
\end{aligned}
$$

Boundary conditions:

$$
\begin{aligned}
& f^{\prime}(x, 0)=f^{\prime}(x, 0)=0 ; h^{\prime}(x, 0)=\frac{x^{\frac{1}{5}} h(x, 0)-1}{1+\gamma x^{\frac{1}{5}} h(x, 0)} \approx-1 \quad(\text { for } x<<1) \\
& f^{\prime}(x, \infty) \rightarrow 0, h(x, \infty) \rightarrow 0
\end{aligned}
$$

Also to find the following for small $x$ equations (16) and (17) become:

$$
C_{f x}=x^{\frac{2}{5}} f^{\prime \prime}(x, 0) \text { and } \theta(x, 0)=x^{\frac{1}{5}} h(x, 0)
$$

For large $x$ those equations (12) and (13) become

$$
\begin{aligned}
& f^{\prime \prime \prime}+\frac{3}{4} f f^{\prime \prime}-\frac{1}{2} f^{\prime 2}+h=x\left(f^{\prime} \frac{\partial f^{\prime}}{\partial x}-f^{\prime \prime} \frac{\partial f}{\partial x}\right) \\
& \frac{1}{\operatorname{Pr}} h^{\prime \prime}+\frac{\gamma}{\operatorname{Pr}} h h^{\prime \prime}+\frac{\gamma}{\operatorname{Pr}} h^{\prime 2}+\frac{3}{4} f h^{\prime}-\frac{1}{5} f^{\prime} h+N x f^{\prime \prime 2}=x\left(f^{\prime} \frac{\partial h}{\partial x}-h^{\prime} \frac{\partial f}{\partial x}\right)
\end{aligned}
$$


Boundary conditions:

$$
\begin{aligned}
& f(x, 0)=f^{\prime}(x, 0)=0 ; h^{\prime}(x, 0)=\frac{h(x, 0)-1}{\{1+\gamma h(x, 0)\} x^{\frac{1}{4}}} \\
& f^{\prime}(x, \infty) \rightarrow 0, h(x, \infty) \rightarrow 0
\end{aligned}
$$

Also to find the following for large $x$ equations (16) and (17) become:

$$
C_{f x}=x^{-\frac{1}{4}} f^{\prime \prime}(x, 0) \text { and } \theta(x, 0)=h(x, 0)
$$

For small $x$, solving equations (18), (19) and (21) together with the boundary condition (20) and for large $x$, solving equations (22), (23) and (25) together with the boundary conditions (24) we will discuss the following results.

\section{Results and Discussion}

The main objective of the present study is to analyze the effect of the thermal conductivity variation due to temperature on natural convective flow along a vertical flat plate in presence of viscous dissipation and the asymptotic solution. In the simulation the values of the Prandtl number $\operatorname{Pr}$ are considered to be $0.73,1.00,1.73,2.97$ and 4.24 that corresponds to hydrogen, steam, water, methyl chloride and sulfur dioxide respectively.

The velocity and the temperature profiles obtained from the solutions of equations (12) and (13) are depicted in Figures 2 to 4. Also the local skin friction and the surface temperature obtained from the solutions of equations (16) and (17) are depicted in Figures 5, 7, 9. Numerical computation are carried out for a range of thermal conductivity variation parameter $\gamma=0.10,0.20$, $0.300 .40,0.50$ and viscous dissipation parameter $N=0.01,0.05,0.10,15,0.20$.

The effect of thermal conductivity variation parameter $\gamma$ on the velocity and temperature against $\eta$ within the boundary layer with $\operatorname{Pr}=1.73$ and $N=0.10$ are shown in figure 2(a) and 2(b), respectively. It is seen from figure 2(a) and 2(b) that the velocity and temperature increase within the boundary layer with the increasing values of $\gamma$. It means that the velocity boundary layer and the thermal boundary layer thickness expand for large values of $\gamma$.

figure 3(a) and 3(b) illustrate the velocity and temperature against $\eta$ for different values of Prandtl number $\operatorname{Pr}$ with $\gamma=0.10$ and $N=0.10$. From fig. 3(a), it can be observed that the velocity decreases as well as its position moves toward the interface with the increasing $P r$. From figure 3 (b), it is seen that the temperature profiles shift downward with the increasing values of $P r$.

In figures 4(a) and 4(b) describe the velocity and temperature against $\eta$ for different values of viscous dissipation parameter $N$ with $\gamma=0.10$ and $P r=1.73$. From figure 4(a), it can be observed that the velocity increases as well as its position moves outward the interface with the increasing values of $N$. From figure 4(b), it is seen that the temperature profiles also the same as increasing 
within the boundary layer. It means that the velocity boundary layer and the thermal boundary layer thickness increase for large values of $N$ from 0.01 to 0.20 .

Figure 5(a) and figure 5(b) illustrate the effect of the thermal conductivity variation parameter on the skin friction coefficient $C_{f x}$ and surface temperature $\theta(x, 0)$ against $x$ with $\operatorname{Pr}=1.73$ and $N=$ 0.10. It is seen from figure 5(a) that the skin friction increases along the upward direction of the plate for a particular value of $\gamma$. It is also seen that the local skin friction coefficient increases for the increasing values of $\gamma$. From figure 5(b), it can be seen that the surface temperature increases monotonically due to the increasing values of $\gamma$ along the positive $x$ direction for a particular $\gamma$.

Figure 7(a) and figure 7(b) deal with the effect of Prandtl number $\operatorname{Pr}$ on the local skin friction coefficient and surface temperature against $x$ with $\gamma=0.10$ and $N=0.10$. It can be observed from figure 7(a) that the skin friction coefficient decreases for a particular value of Pr. It can also be noted that the skin friction coefficient decreases for the increasing values of $P r$. From figure 7(b), it can be seen that the surface temperature decreases monotonically due to the increases along the positive $x$ direction for a particular value of $P r$.

The variation of the local skin friction $C_{f x}$ and surface temperature $\theta(x, 0)$ for different values of $N$ with $\gamma=0.10$ and $\operatorname{Pr}=1.73$ at different positions are illustrated in figures 9(a) and 9(b), respectively. It can also be noted from figure 9(a) that the skin friction coefficient increases monotonically for a particular value of $N$. Again figure $9(\mathrm{~b})$ shows that the surface temperature $\theta(x, 0)$ increases for increasing values of $N$.

Figure 6(a) and 6(b) illustrate the effect of the thermal conductivity variation parameter on the skin friction coefficient $C_{f x}$ and surface temperature $\theta(x, 0)$ for small and large $x$ against $x$ with $\operatorname{Pr}=$ 1.73 and $N=0.10$ for different values of $\gamma$. For small $x$ (for $x<<1$ ) we see that from figure 6(a) the skin friction for values of $\gamma$ almost same. Similarly, for large $x\left(\frac{1}{x}\right.$ as $\left.x \rightarrow 0\right)$, it is observed that the values of $\gamma$ almost similar with all $x$. So the solutions for small and large values of $x$ almost similar with the solution of all values of $x$ near the interface. For small $x$ we see that from figure 6(b) the surface temperature for values of $\gamma$ for all values of $x$ almost same. Similarly for large $x$ it is observed that the values of $\gamma$ for all values of $x$ almost same. There is a divergence near one (1).

Figure 8(a) and figure 8(b) deal with the effect of Prandtl number $\operatorname{Pr}$ on the local skin friction coefficient and surface temperature for small and large $x$ against $x$ with $\gamma=0.10$ and $N=0.10$ for different values of Pr. It can be observed from figure 8(a) that the skin friction coefficient for small $x$ (for $x<<1$ ) of a particular value of $\operatorname{Pr}$ almost same as for all values of $x$. Similarly, for large $x\left(\frac{1}{x}\right.$ as $\left.x \rightarrow 0\right)$, it is observed that the values of skin friction almost similar. From figure 8(b), for small $x$ it can be seen that the surface temperature value of $\operatorname{Pr}$ for all values of $x$ almost same. Similarly for large $x$ it is observed that the values of surface temperature for all values of $x$ almost same. There is a divergence near one (1). 

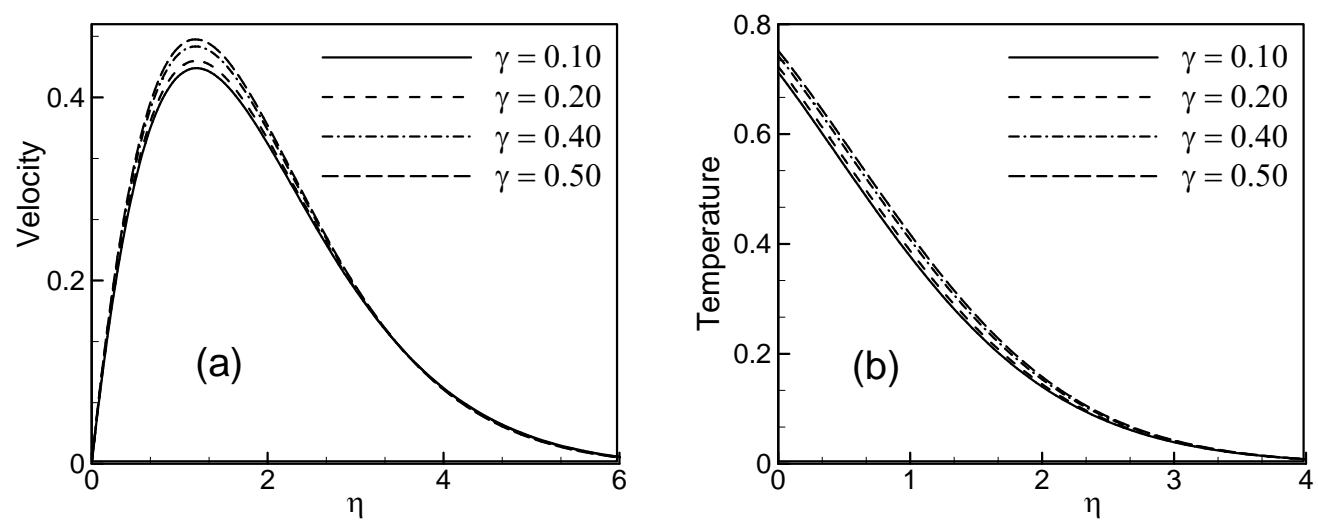

Figure 2(a) Velocity and (b) Temperature against $\eta$ for different values of $\gamma$ with $\operatorname{Pr}=1.73$ and $N=0.10$.
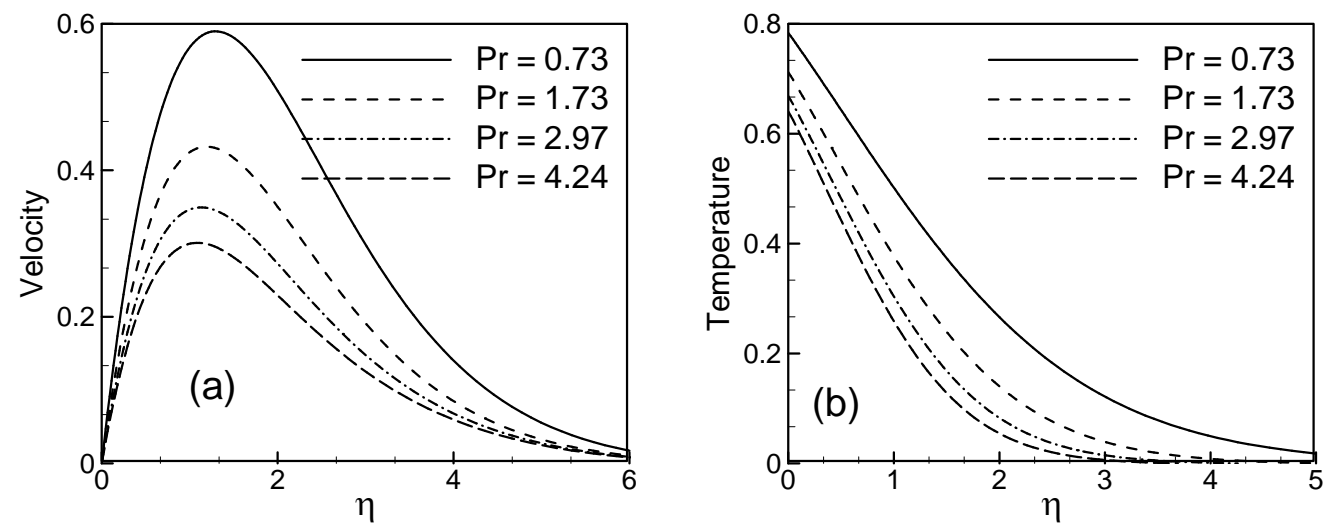

Figure 3(a) Velocity and (b) Temperature against $\eta$ for different values of $\operatorname{Pr}$ with $\gamma=0.10$ and $N=0.10$.
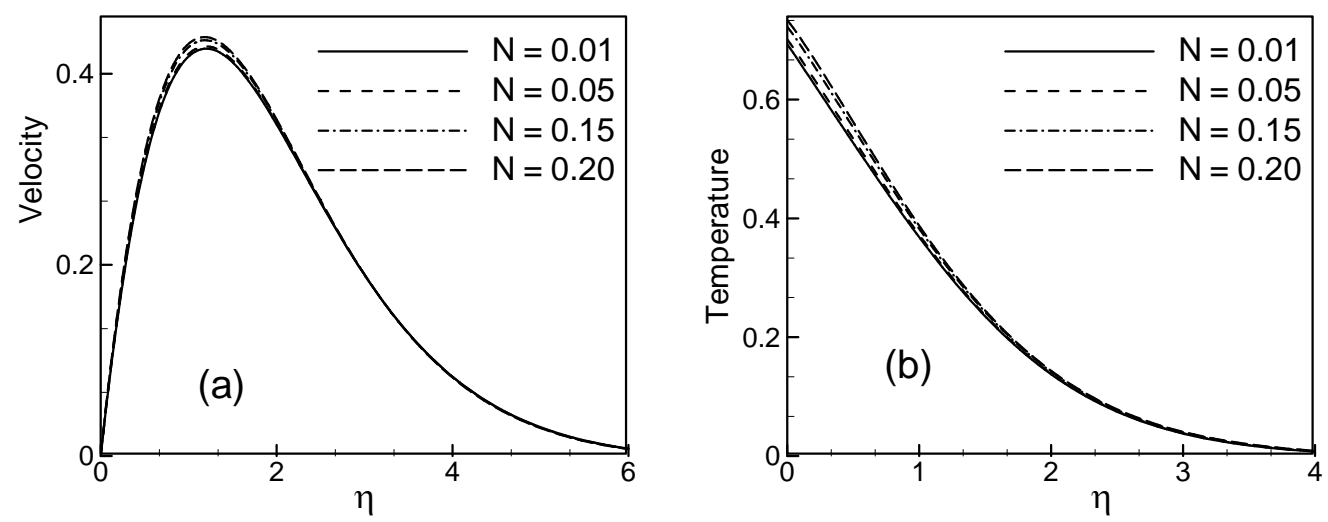

Figure 4(a) Velocity and (b) Temperature against $\eta$ for different values of $N$ with $\gamma=0.10$ and $\operatorname{Pr}=1.73$. 

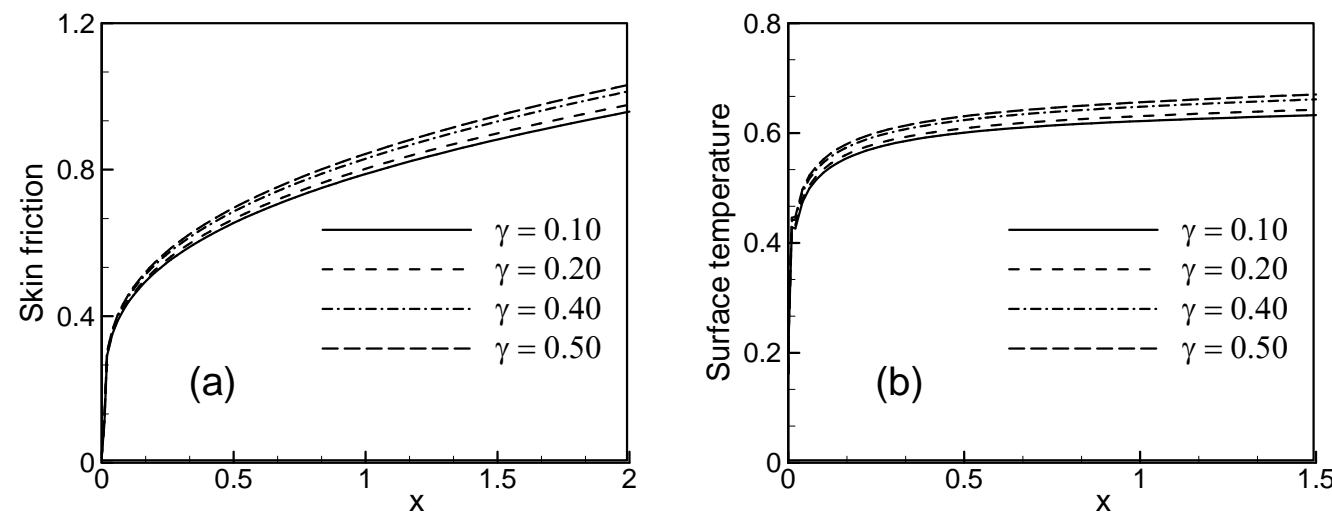

Figure 5(a) Local skin friction coefficient and (b) Surface temperature distribution against $x$ for different values of $\gamma$ with $\operatorname{Pr}=1.73$ and $N=0.10$.
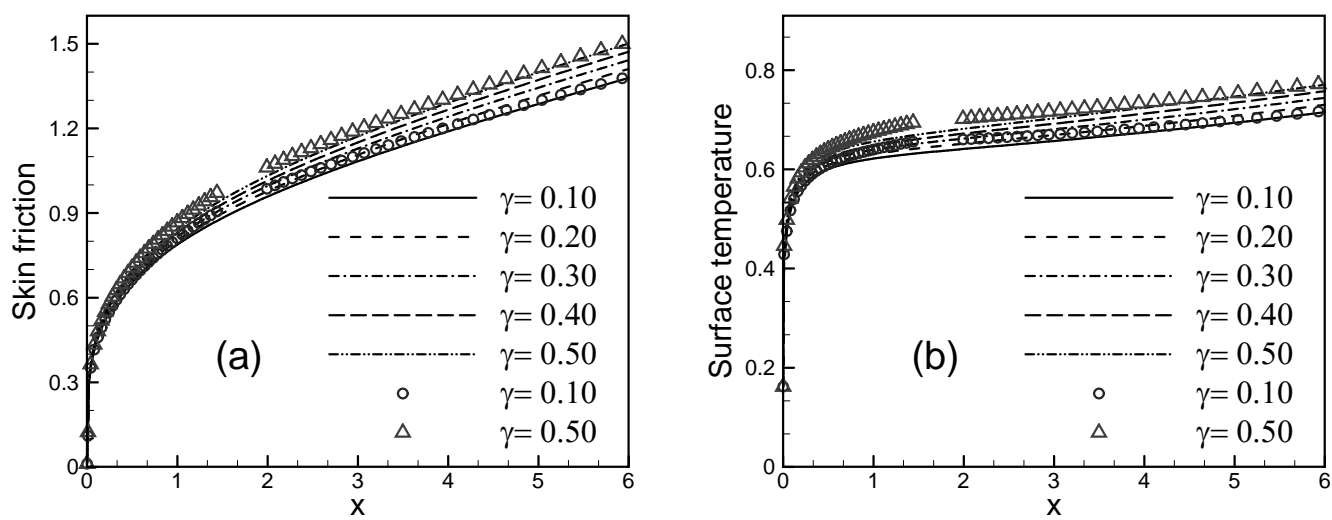

Figure 6(a) Local skin friction coefficient for small \& large $x$ and (b) Surface temperature distribution for small \& large $x$ against $x$ for different values of $\operatorname{Pr}$ with $\gamma=0.10$ and $N=0.10$.
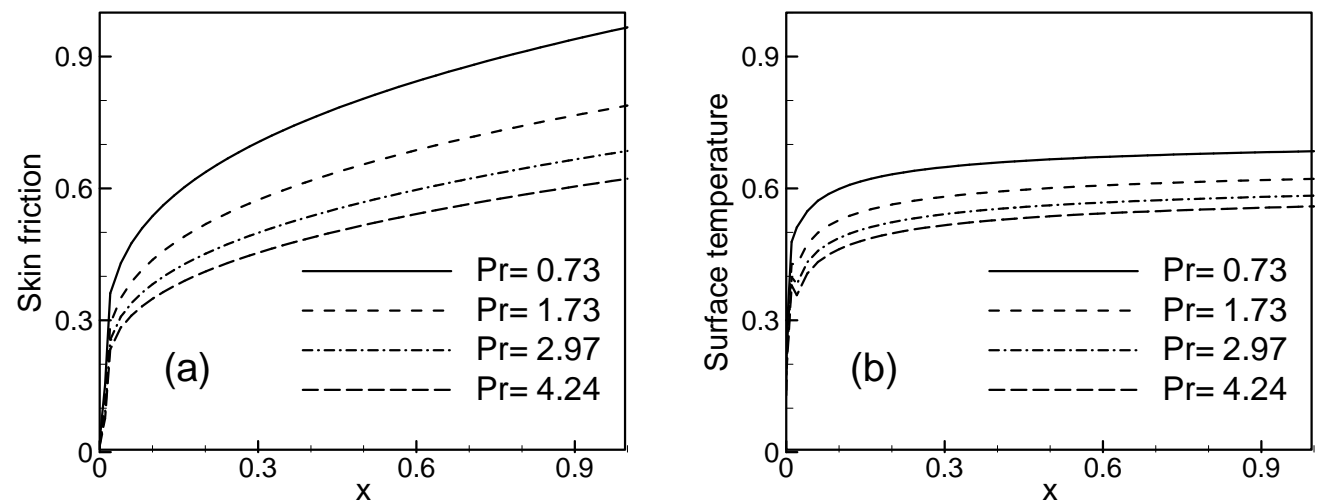

Figure 7(a) Local skin friction coefficient and (b) Surface temperature distribution against $x$ for different values of $\gamma$ with $\operatorname{Pr}=1.73$ and $N=0.10$. 

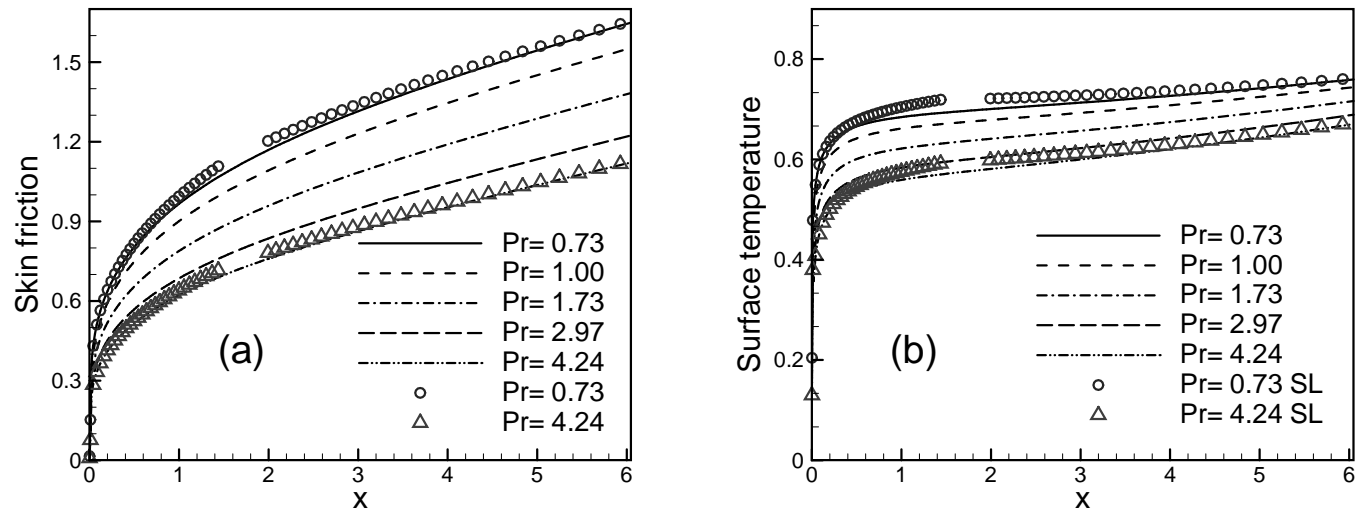

Figure 8(a) Local skin friction coefficient for small \& large $x$ and (b) Surface temperature distribution for small \& large $x$ against $x$ for different values of $\operatorname{Pr}$ with $\gamma=0.10$ and $N=0.10$.
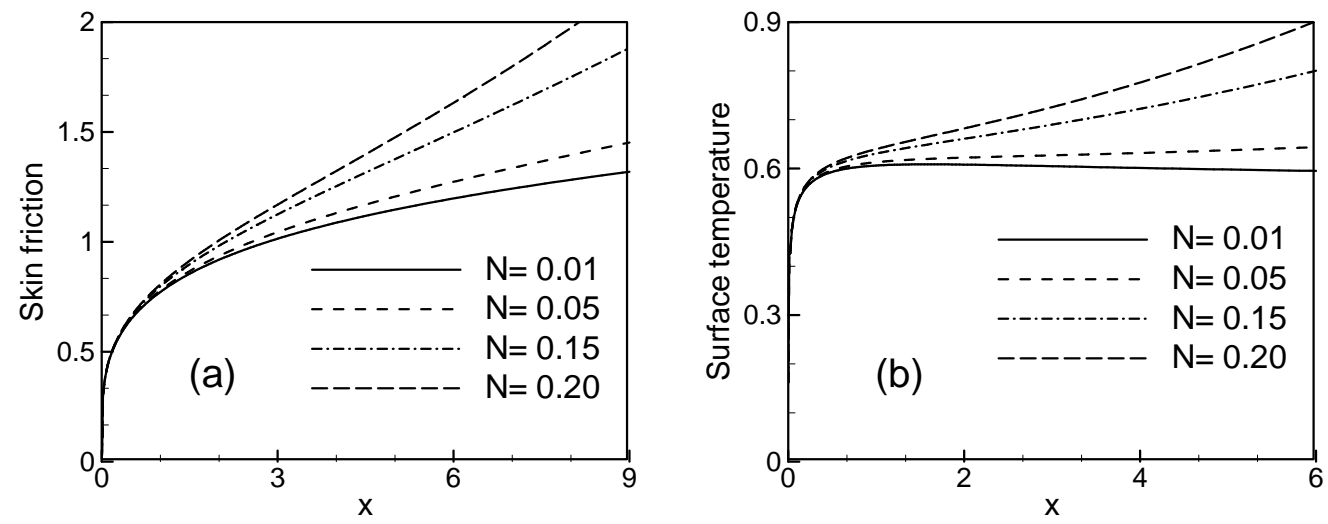

Figure 9(a) Local skin friction coefficient and (b) Surface temperature distribution against $x$ for different values of $N$ with $\gamma=0.10$ and $\operatorname{Pr}=1.73$.
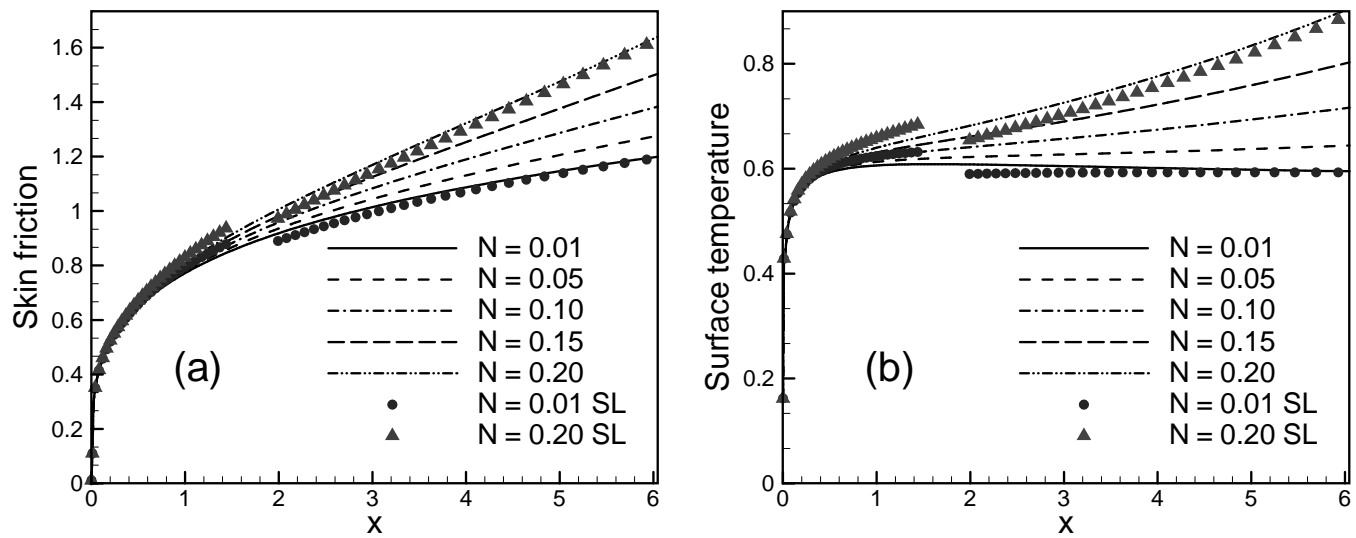

Figure 10(a) Local skin friction coefficient for small \& large $x$ and (b) Surface temperature distribution for small \& large $x$ against $x$ for different values of $N$ with $\gamma=0.10$ and $\operatorname{Pr}=1.73$. 
The variation of the local skin friction coefficient $C_{f x}$ and surface temperature $\theta(x, 0)$ for different values of $N$ for small and large $x$ against $x$ with $\gamma=0.10$ and $\operatorname{Pr}=1.73$ are illustrated in figures 10 (a) and 10(b), respectively. It can also be noted from figure 10(a) that the skin friction coefficient for small $x$ (for $x<<1$ ) of a particular value of $N$ almost same as for all values of $x$. Again the solution for large $x\left(\frac{1}{x}\right.$ as $\left.x \rightarrow 0\right)$ it is observed that the values of skin friction almost similar with all values of $x$. From figure 10(b), for small $x$ it can be seen that the surface temperature value of $N$ for all values of $x$ almost same. Similarly for large $x$ it is observed that the values of surface temperature for all values of $x$ almost same. There is a divergence near one (1).

\section{Comparison of the Results}

Table 1 and Table 2 depict the comparisons of the present numerical results of the skin friction $C_{f x}$ and the surface temperature $\theta(x, 0)$ with those obtained by Pozzi and Lopo (1988) and Merkin and Pop (1996) respectively. Here, the thermal conductivity variation parameter $\gamma$ and viscous dissipation parameter $N$ are ignored (i.e. $\gamma=0$ and $N=0$ ) and the Prandtl number $\operatorname{Pr}=0.733$ with $x^{\frac{1}{5}}=\xi$ is chosen. It is clearly seen that there is an excellent agreement among the present results with the solutions Pozzi and Lopo (1988) and Merkin and Pop (1996).

Table 1: Comparison of the present numerical results of skin friction $C_{f x}$ with Prandtl number $\operatorname{Pr}=0.733$, $\gamma=0$ and $N=0$ against $x$

\begin{tabular}{|c|c|c|c|}
\hline \multicolumn{3}{|c|}{$C_{f x}$} \\
\hline$x^{1 / 5}=\zeta$ & Pozzi and Lupo (1988) & Merkin and Pop (1996) & Present work \\
\hline 0.4 & 0.172 & 0.172 & 0.170 \\
\hline 0.6 & 0.337 & 0.337 & 0.340 \\
\hline 0.7 & 0.430 & 0.430 & 0.423 \\
\hline 0.8 & 0.530 & 0.530 & 0.528 \\
\hline 0.9 & 0.635 & 0.635 & 0.633 \\
\hline 1.0 & 0.741 & 0.745 & 0.748 \\
\hline 1.1 & 0.829 & 0.859 & 0.857 \\
\hline 1.2 & 0.817 & 0.972 & 0.972 \\
\hline
\end{tabular}

Table 2: Comparison of the present numerical results of surface temperature $\theta(x, 0)$ with Prandtl number $\operatorname{Pr}$ $=0.733, \gamma=0$ and $N=0$ against $x$.

\begin{tabular}{|c|c|c|c|}
\hline \multicolumn{3}{|c|}{$\theta(x, 0)$} \\
\hline$x^{1 / 5}=\zeta$ & Pozzi and Lupo (1988) & Merkin and Pop(1996) & Present work \\
\hline 0.4 & 0.493 & 0.493 & 0.481 \\
\hline 0.6 & 0.608 & 0.608 & 0.615 \\
\hline 0.7 & 0.651 & 0.651 & 0.651 \\
\hline 0.8 & 0.684 & 0.686 & 0.687 \\
\hline 0.9 & 0.708 & 0.715 & 0.716 \\
\hline 1.0 & 0.717 & 0.741 & 0.742 \\
\hline 1.1 & 0.699 & 0.762 & 0.763 \\
\hline 1.2 & 0.640 & 0.781 & 0.781 \\
\hline
\end{tabular}

\section{Conclusion}

The effects of surface temperature distribution for the temperature dependent thermal conductivity and viscous dissipation on free convective flow along a vertical flat plate with asymptotic solution 
have been studied in this paper. From the present investigation the following conclusions may be drawn

he velocity within the boundary layer increases for decreasing values of $\operatorname{Pr}$ and for increasing values of $\gamma$ and $N$.

he temperature within the boundary layer increases for increasing values of $\gamma$ and $N$ and decreases for increasing values of $P r$.

he local skin friction coefficient decreases for the increasing values of $P r$ and increases for increasing values of $\gamma$ and $N$.

$\mathrm{n}$ increase in the values of $\gamma$ and $N$ leads to an increase in surface temperature. On the other hand, this decreases for increasing values of $P r$.

n asymptotic solution of $\gamma, \operatorname{Pr}$ and $N$ for skin friction coefficient and surface temperature distribution for small and large values of $x$, there is a good similarity with for all $x$.

\section{REFERENCES}

[1] Miyamoto, M., Sumikawa, J. Akiyoshi, T. and Nakamura, T., (1980). The effect of axial heat conduction in a vertical flat plate on free convection heat transfer, Int. J. Heat Mass Transfer, Vol. 23, No. 11, pp.1545-1553.

[2] Pozzi, A. and Lupo, M., (1988). The coupling of conduction with laminar convection along a flat plate. Int. J. Heat Mass Transfer, Vol. 31, No. 9, pp.1807-1814.

[3] Pop, I. Lesnic, D. and Ingham, D. B. (1995). The conjugate mixed convection on a vertical surface in porous medium, Int. J. Heat transfer, Vol. 38, No. 8 pp.1517-1525.

[4] Gebhart, B. (1962). Effect of dissipation on natural convection, J. Fluid Mechanics, Vol. 14, No. 2, pp. 225-232.

[5] Takhar, H. S. and Soundalgekar, V. M. (1980). Dissipation effects on MHD free convection flow past a semi-infinite vertical plate, Applied Scientific Research, Vol. 36, No. 3, pp. 163-171.

[6] Khan, Z. I. (2002). Conjugate effect of conduction and convection with natural convection flow from a vertical flat plate and in an inclined square cavity, M. Phil thesis, Department of Mathematics, BUET.

[7] Mamun, A. A. (2005). Effects of conduction and convection on magnetohydrodynamic flow with and without dissipation from a vertical flat plate. M. Phil thesis, Department of Mathematics, BUET.

[8] Hossain, M. A. (1992). The viscous and Joule heating effects on MHD free convection flow with variable plate temperature, Int. J. Heat Mass Transfer Vol. 35, No. 12, pp.3485-3487.

[9] Rahman, M. M, Mamun A. A, Azim M. A and Alim, M. A. (2008). Effects of temperature dependent thermal conductivity on magnetohydrodynamic (MHD) free convection flow along a vertical flat plate with heat conduction, Nonlinear Analysis: Modeling and Control, Vol.13, No. 4, pp. 513-524.

[10] Rahman, M. M and Alim, M. A. (2009). Numerical study of magnetohydrodynamic (MHD) free convective heat transfer flow along a vertical flat plate with temperature dependent thermal conductivity, Journal of Naval Architecture and Marine Engineering, JNAME, Vol.6, No.1, pp.16-29.

[11] Nasrin, R. and Alim, M. A. (2009).Combined effects of viscous dissipation and temperature dependent 
thermal conductivity on magnetohydrodynamic (MHD) free convection flow with conduction and joule heating along a vertical flat plate, Journal of Naval Architecture and Marine Engineering, JNAME, Vol.6, No.1, pp.30-40.

[12] Alim, M. A., Alam, Md. M., Mamun A. A. and Hossain, Md. B. (2008). Combined effect of viscous dissipation $\&$ joule heating on the coupling of conduction $\&$ free convection along a vertical flat plate, Int. Commun. In Heat \& Mass Transfer, Vol. 35, No. 3, pp. 338-346.

[13] Alim, M. A., Alam, M. and Mamun, A. A. (2007). Joule heating effect on the coupling of conduction with MHD free convection flow from a vertical flat plate, Nonlinear Analysis: Modeling and Control, Vol.12, No. 3, pp. 307-316.

[14] Merkin J.H and Pop I. (1996). Conjugate free convection on a vertical surface, Int. J. Heat Mass Transfer, Vol. 39, pp.1527- 1534.

[15] Molla M. Md, Rahman A. and Rahman T. L. (2005). Natural convection flow from an isothermal sphere with temperature dependent thermal conductivity, J. Naval Architecture and Marine Engineering, Vol. 2, pp.53-64.

[16] Keller, H. B. (1978). Numerical methods in boundary layer theory, Annual Rev. Fluid Mech. Vol. 10, pp. 417-433.

[17] Cebeci, T and Bradshaw, P. (1984). Physical and Computational Aspects of Convective Heat Transfer, Springer, New York.

[18] Chowdhury, M. K. and Islam, M.N. (2000). MHD Free Convection Flow of Visco-elastic Fluid past an Infinite Porous Plate, Int. J. Heat and Mass Transfer, Vol. 36, No. 5, pp. 439-447, (2000).

[19] Alam, Md. M., Alim, M.A. and Chowdhury, Md. M. K. (2007). Viscous dissipation effects on MHD natural convection flow over a sphere in the presence of heat generation, Nonlinear Analysis: Modeling and Control, Vol.12, No. 4, pp. 447-459.

[20] Saha, S.C., Akter, C. and Hossain, M. A. (2004). Natural Convection from a Plane Vertical Porous Surface in Non-Isothermal Surroundings, Nonlinear Analysis: Modeling and Control, Vol. 9, No. 2, pp.151-170.

[21] Islam Safiqul, A.K.M., Alim, M.A., Karim Rezaul, Md. and Rahman, ATM. M. (2014) Effects of Conduction Variation on MHD Natural Convection Flow Along a Vertical Flat Plate, Bangladesh Journal of Physics, Vol.15, pp. 83-92.

\section{Nomenclature}

$b \quad$ Plate thickness

$C_{f x} \quad$ Local skin friction coefficient

$C_{\mathrm{p}} \quad$ Specific heat at constant pressure

$f$ Dimensionless stream function

$g \quad$ Acceleration due to gravity

Gr Grashof number

$h$ Dimensionless temperature

$l \quad$ Length of the plate

$N \quad$ Viscous dissipation parameter

$P \quad$ Conjugate conduction parameter

$\mathrm{Pr}$ Prandtl number

$T$ Temperature of the interface

$T_{b} \quad$ Temperature at outside surface of the plate

$T_{f} \quad$ Temperature of the fluid

$T_{\infty} \quad$ Temperature of the ambient fluid

$\bar{u} \quad$ Velocity component in $\mathrm{x}$ - direction $\bar{v} \quad$ Velocity component in y- direction

$u \quad$ Dimensionless velocity component in $\mathrm{x}$ - direction

$v$ Dimensionless velocity component in $\mathrm{y}$ - direction

$\bar{x} \quad$ Cartesian co-ordinates

$\bar{y} \quad$ Cartesian co-ordinates

$x \quad$ Dimensionless Cartesian co-ordinates

$y \quad$ Dimensionless Cartesian co-ordinates

\section{Greek Symbols}

$\beta \quad$ Co-efficient of thermal expansion

$\gamma$ Thermal conductivity variation parameter

$\nabla \quad$ Vector differential operator

$\eta \quad$ Similarity variable

$\kappa_{\infty} \quad$ Thermal conductivity of the ambient fluid

$\kappa_{s} \quad$ Thermal conductivity of the solid 
$\kappa_{f} \quad$ Thermal conductivity of the fluid

$\mu \quad$ Viscosity of the fluid

$\mu_{e} \quad$ Magnetic permeability of the fluid

$\checkmark \quad$ Kinematic viscosity $\rho \quad$ Density of the fluid inside the boundary layer

$\sigma \quad$ Electrical conductivity of the fluid

$\tau_{w} \quad$ Shearing stress

$\Psi \quad$ Stream function 